\title{
Influence of the radio signals frequency band on their spatial selection in communication channels
}

\author{
Alexander Okorochkov ${ }^{1, *}$, Nadezda Dmitrienko ${ }^{1}$ \\ ${ }^{1}$ Don State Technical University, Institute of Service and Business (Branch) DSTU in Shakhty, \\ Russia
}

\begin{abstract}
This article deals with the dependence of the signal-to-noise ratio $(\mathrm{S} / \mathrm{N})$ on the frequency band of a radio channel when transmitting three different radio signals over it based on a mathematical model. Signals are transmitted on a single carrier frequency, in one direction and occupy the entire channel frequency band, which varied from 0 to $30 \mathrm{GHz}$. A threeelement sparse antenna array (SAA) is used for signal transmission. Each signal is emitted by all three SAA elements with certain phase shifts. In the reception area, such a structure of the total field is formed, at which the maxima of all transmitted signals are spatially spaced. This allows each signal to be received on a separate antenna. Studies have shown that the $\mathrm{S} / \mathrm{N}$ ratio for different signals depends differently on the channel bandwidth. For a signal emitted by all SAA antennas in phase, the $\mathrm{S} / \mathrm{N}$ ratio is practically independent of the bandwidth and is about $70 \mathrm{~dB}$. For the two remaining phased signals the $\mathrm{S} / \mathrm{N}$ ratio varies equally over the entire range of the channel frequency band values and is characterized by a sharp drop from 62 to $8 \mathrm{~dB}$.
\end{abstract}

\section{Introduction}

Currently, communication systems play an important role in the global economy. Therefore, much attention is paid to their development and improvement and a large number of research papers are devoted to them [1].

Both in the development of new and in the modernization of existing communication systems, it is necessary to take into account many mandatory requirements, among the most important ones are the increase in the speed of information transmission and the rational use of the allocated frequency band [2]. Both of these requirements are contradictory, since in accordance with the Shannon formula, the information transfer rate is directly proportional to the channel bandwidth. This contradiction can be circumvented under multiple use of the frequency band. In this case, the signals transmitted in the same channel frequency band can be separated at reception by some third parameter unique to each signal. Such parameters can be, for example, the time interval of the signal transmission or the code of the digital

\footnotetext{
* Corresponding author: okorochkov a@mail.ru
} 
communication signal, the polarization of the signal field in analogue and digital communication, etc.

In [3], a method for spatial separation of narrow-band signals transmitted in the same direction and at the same carrier frequency $f_{c}=15 \mathrm{GHz}$ was proposed. The essence of the method is that in the area of the receiving antennas for each signal, a unique interference field structure is formed. In it, the amplitude maximum of one of the signals (useful) coincides with the zeros of the amplitude of the other transmitted signals (interference of useful signal) at one spatial point. Placing the receiving antenna at a specified point on the communication range allows receiving a useful signal against the background of interference. The communication quality is characterized by the $\mathrm{S} / \mathrm{N}$ ratio, which can reach more than $60 \mathrm{~dB}$ [4]. The interference amplitude maxima of different signals in space are separated from each other by distances from several meters to several tens of meters. This distance depends on the communication range and the wavelength of the carrier oscillation. Such a distance contributes to the free spatial arrangement of the receiving antennas, in which they do not interfere with each other, and their mutual influence is greatly weakened (with a linear aperture size of up to $1 \mathrm{~m}$ ).

The interference structure of the field of each signal is formed by adding the radiation fields of coherent copies of the signal from the aperture emitters being part of the linear sparse antenna array (SAA). The difference in the interference structures of the fields of different signals was achieved by introducing an individual relative phase shift for each signal between its copies at the inputs of the transmitting antennas. This formation of the radiation fields of different signals allows transmitting on the same carrier frequency with subsequent selection at the reception of up to $\mathrm{N}$ different signals, where $\mathrm{N}$ is the number of emitters in the transmitting SAA [3].

In [5], a method close to the considered one was proposed for spatial selection of various narrowband signals transmitted at one carrier frequency, due to the difference in the curvature of their wave fronts in the reception zone. This difference was ensured by the fact that copies of each signal were emitted by a different number of SAA antennas. However, this method of spatial separation of signals in comparison with the first method has less flexibility in controlling the interference structure of the field in the receiving area and, therefore, provides a lower $\mathrm{S} / \mathrm{N}$ ratio at the reception. Thus in [6] the estimate of the $\mathrm{S} / \mathrm{N}$ ratio for separating signals by the curvature of the wave front shows that this ratio does not exceed $40 \mathrm{~dB}$.

The unifying factor for the considered methods of spatial signal selection is that they are based on more general methods of spatial-temporal signal processing, provided their spatialtemporal narrowband [7, 8]. Modern methods of optimal processing of spatial-temporal signals allow obtaining additional information from radio signals and are widely used in communication systems, passive and active radar systems, remote sensing systems for various purposes $[9,10]$, etc.

Both considered methods of spatial separation of signals are designed for narrow-band signals, i.e. for signals whose bandwidth is much less than the carrier frequency. Therefore, their mathematical models do not take into account the real frequency band. This does not allow investigating the dependence of the efficiency of spatial separation of signals on their frequency band in both methods. Knowing these dependencies is necessary for effective spatial separation of wideband signals, as well as for error estimation for $\mathrm{S} / \mathrm{N}$ ratio, defined with the help of approximate narrowband methods.

The purpose of the work, the results of which are presented in this article, was to study the dependence of the $\mathrm{S} / \mathrm{N}$ ratio on the frequency band of radio signals in the spatial separation of signals according to the first method. The research was carried out on a mathematical model of radio channel signals with internal linear frequency modulation (LFM). 


\section{Construction of mathematical model of the radiation field}

To understand the essence of the research carried out, it is necessary to distinguish between the operating frequency band of the radio channel, which is determined by its amplitudefrequency characteristic (AFC), and the width of the frequency spectrum of the signal. If the channel parameters do not depend on time, then the operating frequency band and AFC remain constant. The spectrum of the information signal, by its nature, is close to the spectrum of a random signal and is a random function of time.

In this paper, we study the dependence of the $\mathrm{S} / \mathrm{N}$ ratio on the channel frequency band, which for a given radio channel does not change over time, but maybe changed when switching to another channel.

The study of the dependence of the $\mathrm{S} / \mathrm{N}$ ratio on the signal spectrum requires a different statistical approach and it was not carried out in this work.

When making up the radio channel model, the following conditions were met:

- the transmitting antenna system was a linear SAA consisting of three circular flat apertures, the centres of which were located on an arc of a circle lying in the horizontal plane, with a radius equal to the communication range $d=50 \mathrm{~km}$, and the distance between the centres of two adjacent apertures $l=14 \mathrm{~m}$ (Fig. 1);

- the radiation field of a circular flat aperture with a radius $a=0.5 \mathrm{~m}$ and uniform amplitude and in-phase excitation of the aperture was chosen as a model of the radiation field of one transmitting antenna SAA;

- the resulting signal field in the receiving area was obtained by scalar summation of the fields of all three antennas emitting identical copies of the signal with the same linear polarization in amplitude;

- the propagation medium of radio waves was considered homogeneous and isotropic, and the propagation conditions in space of the fields of all transmitting antennas were considered the same;

- the carrier frequency of the radiation field was $f_{c}=15 \mathrm{GHz}$ (wavelength $\lambda=2 \mathrm{~cm}$ );

- the multipath nature of radio wave propagation and random errors of the amplitudephase distribution in the aperture of the transmitting SAA were not taken into account;

- the sources of radio emission were considered point-like, due to meet the conditions $a$, $l<<$;

- the spatial structure of the radiation field in the receiving area was analyzed at points of a circular arc with a radius equal to the communication range and with a center coinciding with the geometric center of the transmitting SAA;

- frequency parameters of the channel (carrier frequency, bandwidth, AFC shape) were set by similar parameters of the LFM signal with duration $\mathrm{T}=10^{-6} \mathrm{~s}$, conditionally propagating in the channel; the channel bandwidth corresponds to the total frequency deviation $\triangle f$ of LFM signal.

The choice of the LFM signal for modelling the frequency properties of the channel is explained by two reasons: 1$)$ by a large signal base $B>100(B=\Delta f \cdot T)$ as the signal spectrum has an almost rectangular shape, which facilitates the analysis of processes in the channel; 2 ) the channel bandwidth can be easily adjusted by changing the total deviation of the signal frequency.

The $\mathrm{S} / \mathrm{N}$ ratio in this article refers to the ratio at a given point in the receiving area of the power of the useful signal to the interference power, the role of which is performed by the two remaining signals transmitted in the same channel. So, call this interference as intra systemic. Since this article investigated the influence of only internal interference on the S/N ratio, the noise of the receiving and transmitting equipment, the noise and interference of external sources were not taken into account.If necessary, this noise and interference can be taken into account by adding their power to the intra-system interference. In the general case, 
signals that form intra-system interference carry completely independent information flows, i.e. are random signals in nature. In this work, these signals are uncorrelated. Under this condition, in this work, the total interference power was determined as the sum of the powers of its individual components.

From the above conditions, it follows that both centres of the transmitting antennas of the SAA and the centres of the receiving antennas are located on the arcs of circles with the same radius equal to the communication range. With this arrangement of the receiving antennas, a higher $\mathrm{S} / \mathrm{N}$ ratio is achieved than with their rectilinear arrangement [11]. The symmetrical arrangement of the receiving and transmitting antennas on the arcs of the circles (Fig. 1) simplifies the technical implementation of radio channels with spatial separation of signals in radio relay communication.

Fig. 1 shows a block diagram of a communication system with spatial separation of signals based on an emitting SAA with three emitters in a polar coordinate system. The centre of the polar coordinate system is aligned with the point A0, the polar axis coincides with the line $\mathrm{A} 0-\mathrm{B} 0$, and the direction of the positive values of the angle $\theta$ is indicated by the arrow at the point $\mathrm{B} 0$. At points $\mathrm{A} 1, \mathrm{~A} 0$ and $\mathrm{A} 2$ on the left dashed arc, the phase centres of the transmitting antennas of the SAA are located, which in the future we will be denoted as these points. The directions of the main beams of the radiation patterns of all transmitting antennas are installed parallel to the A0-B0 line. The field in the receiving area will be investigated at the points of the right dotted arc (point $\mathrm{D}$ is one of them). The communication range (length of the A0-B0 line) is designated by the letter $d$. The right and left arcs have the same radii equal to $d$, and their centres are located at points $\mathrm{A} 0$ and $\mathrm{B} 0$, respectively. The meaning of the remaining linear and angular quantities is clear from Fig. 1.

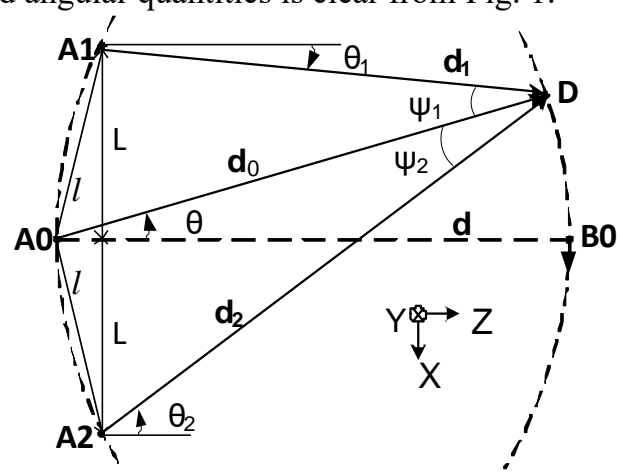

Fig. 1. Block diagram of a communication system with spatial separation of signals

When constructing the SAA radiation field model, this field was defined as the scalar sum of the fields of individual emitters with the same polarization. With the accepted in the article the carrier frequency of the radiation field $f_{c}=15 \mathrm{GHz}(\lambda=2 \mathrm{~cm})$ and the radius of the circular aperture $a=0.5 \mathrm{~m}$, the lower boundary of the radiation zone is $100 \mathrm{~m}$. Since the communication range is assumed to be $d=50 \mathrm{~km}$, then to construct a model of the radiation field of a separate SAA emitter, you can use the normalized amplitude radiation pattern $\mathrm{F}$ $(\theta, \omega)$ of a circular aperture with equal-amplitude and in-phase excitation

$$
F(\theta, \omega)=\frac{1+\cos (\theta)}{2} \cdot \frac{J_{1}[a \omega \sin (\theta) / c]}{a \omega \sin (\theta) / c}
$$

where $J_{1}(\ldots)$ - is Bessel function of the first kind of the first order, $c$ - is the speed of electromagnetic waves in free space, $\omega-$ is circular frequency. Taking into account the decision taken, the radiation field of a separate aperture emitter carrying a LFM pulse can be represented as following: 


$$
E_{n}=E_{m, n} \cdot \frac{\omega_{n}}{2 \pi c d_{n}} \cdot F\left(\theta_{n}, \omega_{n}\right) \cdot \exp \left(i \Phi_{n}\right)
$$

Where $n$-is an integer subscript for the field parameter, which takes the values $0,1,2$ and denotes the parameter belonging to the field of one of the three SAA antennas (the index $n$ coincides with the second digit in the designations of antennas A0, A1, A2); $E_{m, n}$ - is the amplitude factor that takes into account the signal strength at the antenna input and the antenna gain; $\Phi_{n}-$ is a phase characteristic of the LFM signal field.

The phase characteristic of the field $\Phi_{n}$ in (2) has the form of the phase of the LFM pulse

$$
\Phi_{n}=\omega_{c}\left(t-t_{d, n}\right)+\frac{\Delta \omega}{2 T}\left(t-t_{d, n}\right)^{2}
$$

where $\omega_{\mathrm{c}}=2 \pi f_{\mathrm{c}}, \Delta \omega=2 \pi \Delta f, t_{d, n}$ - is the signal delay time during propagation from the $n$ th emitter to the point of reception, determined by the ratio $t_{d, n}=$ $d_{n} / c$ (distances $d_{n}$ marked on Fig. 1 ), $t$ - is current time counted from the moment of signal emission. The signal delay time $t_{d, n^{-}}$is determined between the moments when half of the pulse width passes through the transmitting and receiving apertures. The current frequency of the LFM pulse within the interval of its duration

$$
t_{d, n}-\frac{T}{2} \leq t \leq t_{d, n}+\frac{T}{2}
$$

is determined by the known relation

$$
\omega_{n}=\omega_{\mathrm{c}}+\Delta \omega \frac{t-t_{d, n}}{T}
$$

To simplify the mathematical calculations when obtaining the final expression for the model of the total signal field in the receiving area, some explanatory relations that follow from Fig. 1 can be written:

$$
\theta_{1}=\theta+\psi_{1}, \quad \theta_{2}=\theta-\psi_{2}, \quad d_{0}=d, \quad t_{d, 0}=d / c
$$

As will be seen later, the analysed range of angles $\theta$ is approximately $2 \cdot 10^{-3} \mathrm{rad}$, so all terms above the first order of the angle $\theta$ can be ignored, which leads to the following approximate relations:

$$
\begin{gathered}
l \approx L, d_{1} \approx d+L \cdot \theta, d_{2} \approx d-L \cdot \theta, t_{d, 1} \approx(d+L \cdot \theta) /{ }_{c}, t_{d, 2} \approx(d-L \cdot \theta) /_{c} \\
\Phi_{1} \approx \Phi_{0}-\omega_{0} L \theta / c, \quad \Phi_{2} \approx \Phi_{0}+\omega_{0} L \theta / c
\end{gathered}
$$

where the phase $\Phi_{0}$ is determined by formula (3) at $n=0$.

Let us make the last simplifying assumption. For small values of the angle $\theta$, the three first amplitude factors on the right side of (2) for different copies of the same signal are practically the same. We assume that the values of these factors are equal to their values for the central antenna A0 in Fig. 1, and introduce a notation for their product $A\left(\theta, \omega_{0}\right)$. Then, taking into account (6) and (7), the final expression for the total field of a single LFM signal emitted by all the SAA antennas at the receiving point will have the form:

$$
E_{\Sigma} \approx A\left(\theta, \omega_{0}\right) \cdot \exp \left(i \Phi_{0}\right) \cdot\left[1+2 \cos \left(\omega_{0} L \theta / c\right)\right]
$$


To estimate the $\mathrm{S} / \mathrm{N}$ ratio, it is necessary to know the coordinates of zeros in the distribution of the field amplitude $E_{\Sigma}$ along the arc of the circle at the distance of the communication range. Equating the modulus of the right-hand side in (8) to zero, we obtain an equation for determining the desired coordinates

$$
1+2 \cos \left(\omega_{0} L \theta / c\right)=0 .
$$

The set of solutions to this equation has the form

$$
\theta_{0}(t)=\frac{c}{\omega_{0}(t) L} \cdot\left( \pm \frac{2}{3} \pi+2 \pi k\right), \quad k=0, \pm 1,2 \ldots
$$

As follows from (10), taking into account (5), the coordinates of the zeros of the full field amplitude depend on the time, frequency deviation, and duration of the LFM pulse. Under the condition $\Delta f=0$, the expression (10) turns into a similar expression for the zeros of the narrow-band signal amplitude, obtained in [3].

Fig. 2 shows the angular distributions of the fields of the three LFM signals, calculated by the formula (8) for the time moment $t=t_{d, 0}=d / c$. Since at any time within the interval of the LFM pulse duration, its spectrum consists of only one frequency, all the fields in Fig. 2 correspond to single-frequency signals with frequencies $\omega \cong \omega_{c}$. The solid red line is a graph obtained by summing at the point of analysis of in-phase copies of the signal from three transmitting antennas. When plotting the graph, indicated by a brown dash-dotted line, the signals at the inputs of antennas A1 and A2 were given phase shifts $\varphi_{2}=-2 \pi / 3$ и $\varphi_{3}=$ $2 \pi / 3$ relative to the signal at the input of antenna $A 0$, respectively. The graph shown by the blue dashed line was built similarly to the previous graph, taking into account the change in the signs of the phase shifts to opposite ones. The legend in Fig. 2 shows the phase shifts for all signals at the input of antenna A1. In accordance with the plotting conditions, the field and the signal $\mathrm{S} 1(\theta)$ corresponding to the red graph are called in-phase, and the other two fields and the corresponding signals $\mathrm{S} 2(\theta)$ and $\mathrm{S} 3(\theta)$ are called phased.

In Fig. 2 by choosing the phase shifts $\varphi_{2,3}$, the maxima of the amplitudes of the useful signals were combined with the zeros of the amplitudes of the interference signals, which ensures a high $\mathrm{S} / \mathrm{N}$ ratio. Since these conditions are repeated when moving to the right and left along the $\theta$ angle axis from the origin of coordinates, then in the future we will analyze the region of angles, which includes three main maxima: in-phase and two phased signals, most closely located to the origin.

The fields described by formula (8) have two serious drawbacks that do not allow them to be used directly to study the influence of the frequency band (frequency deviation) LFM signal on the $\mathrm{S} / \mathrm{N}$ ratio. The first drawback is the time dependence of the field represented by formula (8), which makes it difficult to analyse it. The second drawback is that the field in (8) is single-frequency at each time, as the LFM signal itself. That is, it does not carry information about the spectrum of the LFM signal as a whole for the entire interval of the signal duration $T$.

The noted drawbacks can be eliminated if, instead of the field (8), we consider its average value over the interval of the LFM signal duration. This averaging is carried out in accordance with the formula 


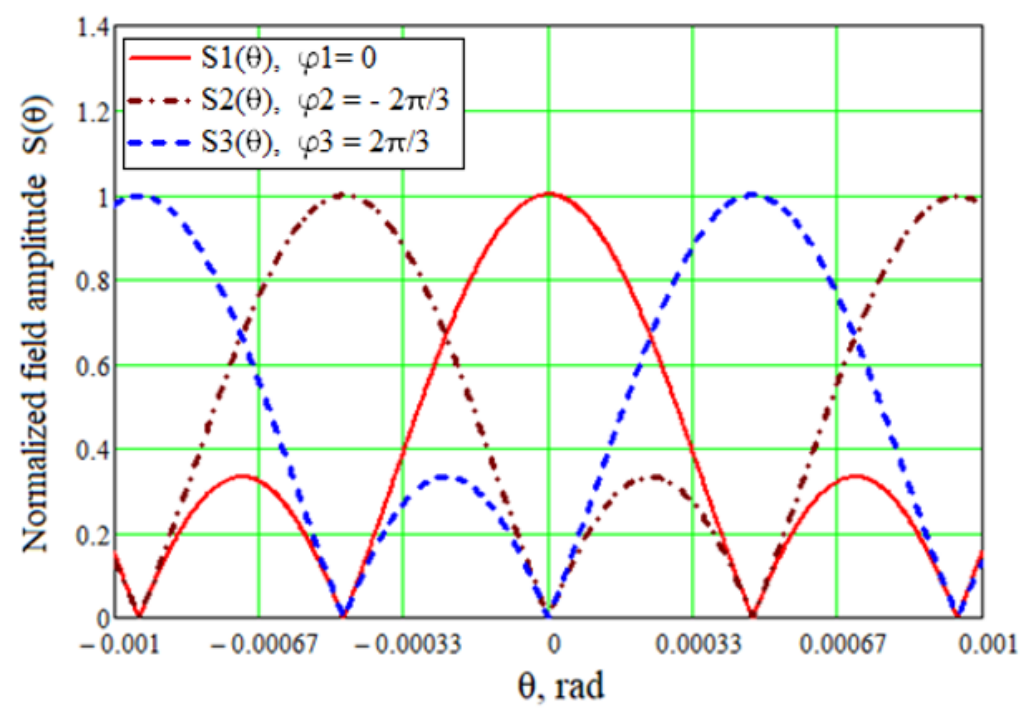

Fig. 2. Angular distribution of the fields of three LFM signals with different relative phase shifts between their copies at the inputs of different transmitting antennas

$$
E_{\tau, p}(\theta)=\int_{t_{d, 2}-0.5 T}^{t_{d, 1}+0.5 T} \frac{E_{\Sigma \mathrm{p}}(t, \theta)}{E_{\Sigma 1}(t, 0)} d t / \tau(\theta)
$$

where $\tau(\theta)$ - is the time interval during which all three copies of one signal are present on the receiving antenna aperture; $p$ - is a signal number ( $p=1$ for in-phase signal, $p=2,3$ are for phased signals); $E_{\Sigma 1}(t, 0)$ - is the field of the first (in-phase) signal in the direction $\theta=0$ (normalizing factor). The time interval $\tau(\theta)$ is calculated by the formula

$$
\tau(\theta)=t_{d, 1}(\theta)+T-t_{d, 2}(\theta)
$$

Thus, because of the integration operation, the resulting field $E_{\tau, p}(\theta)$ does not depend on time and has the entire spectrum of the LFM pulse. Therefore, this field can be taken as the field of the reference model signal, which does not change in time over the duration of the LFM pulse and has a constant rectangular spectrum. The width of this spectrum can be adjusted in the range from zero to twice the value of the carrier frequency of the LFM pulse. Such a signal is very well suited for studying the dependence of the $\mathrm{S} / \mathrm{N}$ ratio on the bandwidth of the radio channel frequencies.

\section{Investigation of the influence of the radio signals frequency band of on the quality of their spatial separation}

Using the constructed signal model (11), the influence of its frequency band on the angular distribution of the radiation field amplitude in the receiving region was studied. The aim of the study was to determine the factors affecting the quality of the spatial separation of signals, i.e. decreasing the $\mathrm{S} / \mathrm{N}$ ratio. For this, using (11), angular distributions of the amplitudes of the radiation fields of in-phase and two phased signals for different bandwidths of their frequency bands were constructed and their comparative visual analysis was carried out. The frequency bands were the same for all signals. The relative phase shifts between the copies of the phased signals were taken the same as for the signals in Fig. 2. The field amplitude was determined as the modulus of the expression on the right side of formula (11). 
Figs. $3-5$ show the most characteristic graphs of the angular distributions of the amplitudes of the radiation fields of the in-phase and two phased signals for different values of their bandwidth taken from the range $0<\Delta f \leq 2 f_{c}$. The graphs of the corresponding signals are constructed with the same lines as in Fig. 2. For a comparative analysis of changes in the angular distributions of the field amplitudes during the expansion of the signal frequency band, the graphs in Fig. 2 were used as reference graphs, since they correspond to single-frequency signals, i.e. signals with zero frequency band. The area of angles on the graphs, which includes three main central maxima corresponding to three different signals, was analyzed.

The results of the visual analysis of the graphs are as follows. With a zero frequency band (Fig. 2), the main maxima of all three signals have the same values and the coordinates of "zero" amplitudes of the interference signals coincide with the coordinates of the corresponding maxima. The values of the signals at the points of the amplitude "zeros" are actually not equal to zero, but have some minimum values due to the inaccurate determination of the coordinates of the "zeros" graphically. In addition, the S/N ratio is estimated not by the signal levels at a certain point, but by the signals at the output of the receiving antenna with a finite in area aperture. Both noted factors significantly reduce the $\mathrm{S} / \mathrm{N}$ ratio. However, it still remains very high, and according to the parameters adopted in this work, its calculated value exceeded $70 \mathrm{~dB}$.

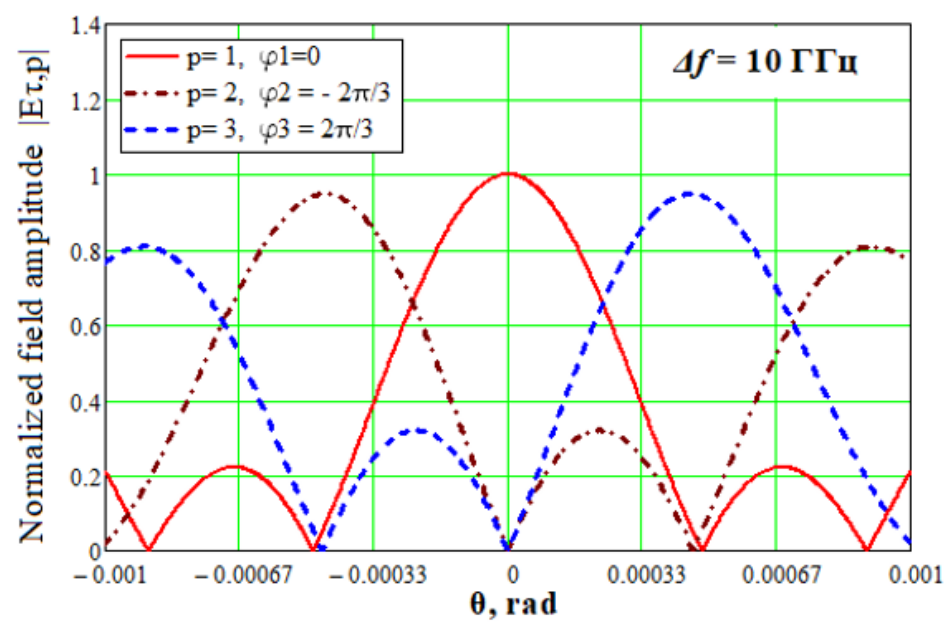

Fig. 3.Angular distribution of the fields of the in-phase signal $(p=1)$ and two phased signals $(p=2,3)$ for the frequency bandwidth $\Delta f=10 \mathrm{GHz}$

According to the results of the analysis of the graphs in Figs. 3 - 5 when expanding the frequency band, the following conclusions can be made:

- the position and level of the central maximum of the in-phase signal and the corresponding "zeros" of the interference amplitude from phased signals practically do not change in the entire used range of frequency band values; this leads to an insignificant dependence of the $\mathrm{S} / \mathrm{N}$ ratio on the frequency band for the in-phase signal;

- the levels of the maxima of the phased signals decrease, and the corresponding "zeros" of the amplitudes of the interference signals are shifted relative to each other in 


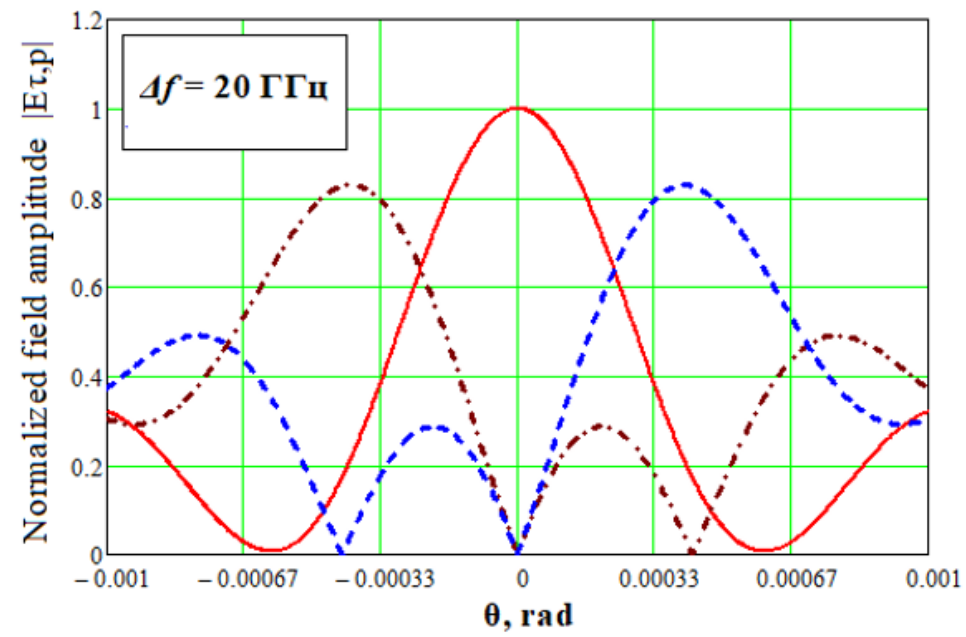

Fig. 4.Angular field distribution of the in-phase signal and two phased signals for a bandwidth of $\Delta f=20 \mathrm{GHz}$

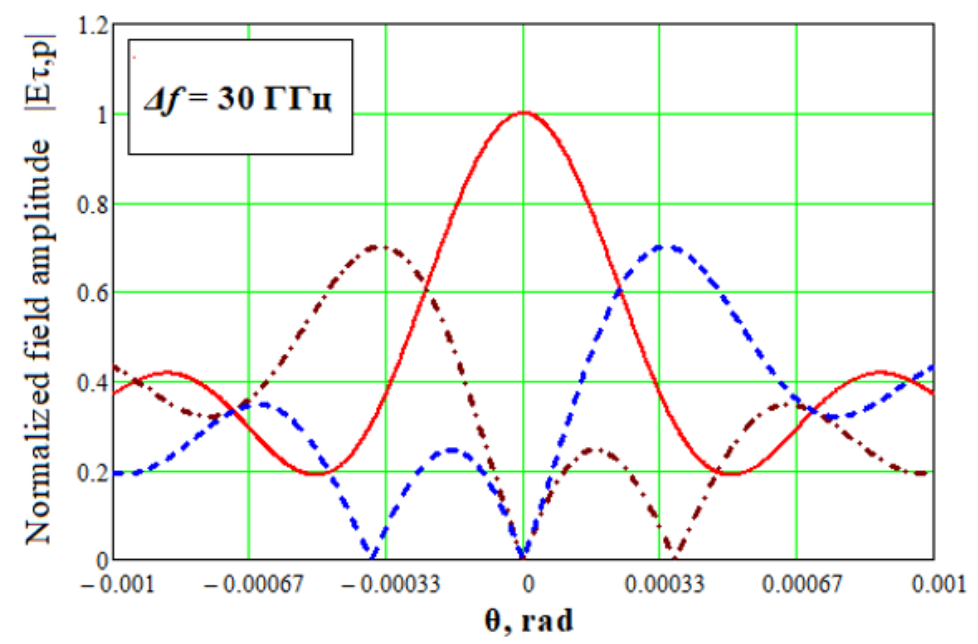

Fig. 5.Angular field distribution of the in-phase signal and two phased signals for a bandwidth of $\Delta f=30 \mathrm{GHz}$

different directions; this leads to the fact that in the total interference signal "zeros" turn into minima, the level of which increases with the expansion of the frequency band; all this leads to a decrease in the $\mathrm{S} / \mathrm{N}$ ratio when receiving phased signals.

The analysis of the graphs in Figs. 4, 5 shows that the structure of the field of the in-phase signal in the region of its first minima from the origin of coordinates changes most of all with an increase in the frequency band. The level of the field of this signal in the region of the maxima of the field of phased signals creates great interference for these signals and significantly reduces the quality of their spatial separation during reception. There are two ways to improve the quality of phased signal reception:

- to exclude the transmission of in-phase and phased signals in the same frequency band;

- to transmit the in-phase signal with field polarization orthogonal to the field polarization of phased signals. 
The first method is accompanied by a decrease in the information transfer rate and therefore is not always acceptable.

The second method is usually used in communication systems with polarizing channel densification. However, the receiving antennas are required to suppress the cross-polarization component by at least $30 \mathrm{~dB}[12]$.

In conclusion, on the basis of the conducted studies, the graphs of the dependence of the $\mathrm{S} / \mathrm{N}$ ratio on the bandwidth for different signals were calculated and constructed, as shown in Fig. 6.

When writing the formula for calculating and plotting the dependence of the $\mathrm{S} / \mathrm{N}$ ratio on the signal bandwidth, the following assumptions were made:

- the phase centre of the receiving antenna was located at "zero" or minimum amplitude of the total interference signal corresponding to the maximum amplitude of the received useful signal; since the coordinates of the "zeros" or minima of the amplitude of the interference signals changed when the frequency band is changed, it was necessary to adjust the position of the phase centre of the receiving antenna each time;

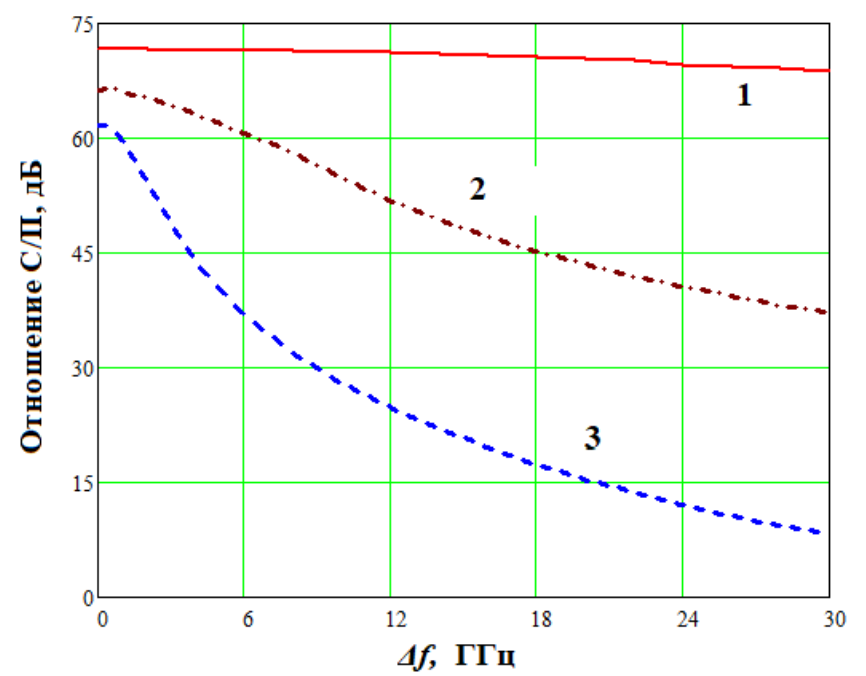

Fig. 6. Graphs of the dependence of the $\mathrm{S} / \mathrm{N}$ ratio on the bandwidth for: 1 - in-phase signal; 2 - phased signal with ain-phase signal suppressed by $30 \mathrm{~dB} ; 3$ - phased signal

- the conditional receiving antenna aperture had the shape of a square with a side $1 \mathrm{~m}$; due to the horizontal arrangement of the transmitting antennas, it was believed that the interference structure of the field is most pronounced only in the horizontal plane (XZ plane in Fig. 1), and in the vertical plane (YZ plane in Fig. 1) the field at the receiving aperture was considered unchanged; therefore, the signal level at the output of the receiving antenna was determined by integrating the signal field over the angle $\theta$ only along the horizontal size of the aperture.

Taking into account the accepted assumptions, the formula for calculating the $\mathrm{S} / \mathrm{N}$ ratio in the case of in-phase signal takes the following form:

$$
S / N=10 \log \left[S S_{1} /\left(S S_{2}+S S_{3}\right)\right]
$$

where $S S_{p}=\left|\int_{\theta 01-\beta}^{\theta 01+\beta} E_{\tau, p}(\theta) d \theta\right|^{2}-$ is a coefficient which is proportional to the power of the in-phase signal at $p=1$, and proportional to the power of the interference signal at $p=2$, 
3; $\theta_{01}$ - "zero" coordinate of the noise-signal amplitude, corresponding to the maximum of the in-phase signal; $\beta$ - half the angle at which the horizontal side of the receiving aperture is visible from the phase center of the transmitting SAA.

Based on (12), a formula can be obtained for calculating the $\mathrm{S} / \mathrm{N}$ ratio for a phased signal by cyclically rearranging the lower numeric indices on the right side of the formula (12) and replacing in all integrals in (12) "zero" coordinate of the interference signal amplitude for inphase signal by a similar value for the corresponding phased signal.

The formulas obtained in this way were used to calculate and plot the graphs in Fig. 6. The graph number 1 corresponds to the in-phase signal. As predicted earlier, the $\mathrm{S} / \mathrm{N}$ ratio for this signal is very weakly dependent on the bandwidth and reaches its maximum values under the conditions of the conducted studies.

Fig. 6, number 3, shows a graph for only one of the phased signals, since both phased signals are equivalent in their properties. In this case, the $\mathrm{S} / \mathrm{N}$ ratio drops quite strongly with the increase in the signal bandwidth. The reasons for this were discussed above when analysing the graphs in Figs. 3 - 5. For phased signals, the width of the operating frequency band is determined by the minimum allowable level of the $\mathrm{S} / \mathrm{N}$ ratio. Thus, with a minimum $\mathrm{S} / \mathrm{N}$ level of $30 \mathrm{~dB}$, the bandwidth of the phased signal reaches $9 \mathrm{GHz}$. In general, this is also a good result, although it is inferior to the in-phase signal.

Graph number 2 takes the middle position between graphs 1 and 3 and corresponds to a phased signal, provided that the interfering signal is the sum of the interference from the second phased signal and the $30 \mathrm{~dB}$ suppressed interference from the in-phase signal. Inphase suppression occurs due to the use of the cross-polarization properties of the receiving antenna when transmitting in-phase and phased signals at orthogonal polarizations. In this case, there is a slower decline in the $\mathrm{S} / \mathrm{N}$ ratio with an increase in the signal frequency band due to the actual elimination of one of the two interferences. In graph 2 , the $\mathrm{S} / \mathrm{N}$ ratio does not fall below $37 \mathrm{~dB}$ even at the maximum signal bandwidth, which is sufficient for most types of signals transmitted in communication channels.

It was assumed above that the interference signals are not correlated with each other. The correlation existence between these signals in the worst case can lead to $3 \mathrm{~dB}$ decreasing of $\mathrm{S} / \mathrm{N}$ ratio.

\section{Conclusion}

Earlier in works $[3,4,11]$ to increase the speed of information transmission and efficient use of the frequency resource was proposed and investigated communication system with spatial separation of signals for the transmission of narrowband signals.

In this paper, as a result of the research conducted on mathematical models of LFM signals, it was shown that the proposed communication system can also be used to transmit broadband signals with high efficiency of their spatial separation, estimated by the level of $\mathrm{S} / \mathrm{N}$ at the output of the receiving antenna.

In the work performed, a communication system based on a transmitting SAA, including three aperture antennas, was investigated. As a result of the conducted research, it was found that the transmission of broadband signals in communication systems with their spatial separation has the following features:

- all three transmitted signals by their properties can be attributed to two groups, one of which is the in-phase signal, and the other two phased signals;

- the spatial structure of the field of the in-phase signal in the receiving area is formed when its three identical copies are transmitted by three SAA antennas (Fig. 3); the S/N ratio for this signal very weakly depends on its bandwidth in the entire investigated range (from 0 to $30 \mathrm{GHz}$ ) and reaches over $70 \mathrm{~dB}$ (Fig. 6); this signal is the most protected from intrasystem interferences; 
- the spatial structure of the field of each of the phased signals is formed when three copies of each signal are transmitted by three SAA antennas with additional individual phase shifts between the copies installed at the inputs of the transmitting antennas (Fig. 3); the S/N ratio for these signals decreases rapidly from 62 to $8 \mathrm{~dB}$ as the bandwidth expands from 0 to $30 \mathrm{GHz}$ (Fig. 6); these signals are the weakest protected from intra-system interference, their frequency band is determined by the permissible minimum level of the $\mathrm{S} / \mathrm{N}$ ratio;

- the degree of protection of each of the phased signals from intra-system interference can be significantly increased by transmitting these signals and the in-phase signal using fields with mutually orthogonal polarizations, followed by suppression of the in-phase crosspolarization signal due to the polarization selectivity of the receiving antenna; with a polarization suppression of interference from the in-phase signal by $30 \mathrm{~dB}$, the level of the $\mathrm{S} / \mathrm{N}$ ratio in the entire investigated range of values of the frequency band of the phased signal increases by 4.6 ... $29 \mathrm{~dB}$ (Fig. 6).

The presented work is a continuation of the previously completed initiative work by the author together with his students on the study of communication systems with spatial separation of signals. Further research will be aimed at finding opportunities to ensure equally high security of all transmitted signals from intra-system interference, studying the features of transmitting a larger number of signals at one carrier frequency, finding ways to reduce the harmful effect of multipath signal propagation, developing individual devices for the practical implementation of the studied communication system with spatial separation signals, taking into account the influence of the shape of the signal spectrum on the quality of their spatial separation at the reception, as well as on the solution of other problems that may arise as the result of the abovementioned studies.

\section{References}

1. H. Tataria, M. Shaf, A.F. Molisch, M. Dohler, H. Sjöland, F. Tufvesson, Proc. of the IEEE (Early Access) (Mar. 2021), p.p. 1-34

2. M. Lamba, Ch. Singh, SOP Transactions on Wireless Communications, 1, 2, 42 (2014)

3. A.I. Okorochkov, B.B. Malyutin, Serbian journal of electrical engineering, 16, 211 (2019)

4. A. I. Okorochkov, B. B. Malyutin, M. A. Laouar, N. A. Dmitrienko, E3S Web of Conferences 104, 02005 (2019)

5. Ye. A. Yakornov, M. A. Kolomytsev, H. L. Avdieienko, O. Y. Lavrinenko, Visnyk NTUU KPI Seriia - Radiotekhnika. Radioaparatobuduvannia (Ukraine), 48, 97 (2012)

6. H. L. Avdienko, Ye. A. Yakornov, Visnyk NTUU KPI Seriia - Radiotekhnika. Radioaparatobuduvannia (Ukraine), 52, 92 (2013)

7. I. Ya. Kremer, A. I. Kremer, V. M. Petrov, V. A. Pon'kin, N. A. Potapov, Space-Time Signal Processing, Moscow: Radio and communication (1984)

8. R. Klemm, Applications of Space-Time Adaptive Processing ed., London: IEE (2004)

9. V.F. Kravchenko, D.V. Churikov, J. Antennas: p.h. Radiotekhnika (Russia), 4 (131), 47 (2008)

10. V. K. Volosuk, Yu. V. Gulyaev, V. F. Kravchenko, B. G. Kutuza, V. V. Pavlikov, V. I. Pustovoit, J. Radio Engineering and Electronics: p.h. Akademkniga (Russia), 59, 2, 109 (2014) 
11. A. I. Okorochkov, B. B. Malyutin, G. L. Khripkov, M. A. Laouar, A. A. Priezhev, AIP, 2188, 050002 (2019)

12. O.P. Frolov, Antennas for satellite earth stations, Moscow: Radio and communication (2000) 